\title{
Wall slip of complex fluids: interfacial friction versus slip length
}

\author{
Benjamin Cross, Chloé Barraud, Cyril Picard, and Élisabeth Charlaix** \\ Univ. Grenoble Alpes, CNRS, LIPhy, F-38000 Grenoble, France. \\ Liliane Léger and Frédéric Restagno \\ Laboratoire de Physique des Solides, CNRS, Université Paris-Sud, \\ Université Paris-Saclay, 91405 Orsay Cedex, France
}

\begin{abstract}
Using a dynamic Surface Force Apparatus, we demonstrate that the notion of slip length used to describe the boundary flow of simple liquids, is not appropriate for viscoelastic liquids. Rather, the appropriate description lies in the original Navier's partial slip boundary condition, formulated in terms of an interfacial friction coefficient. We establish an exact analytical expression to extract the interfacial friction coefficient from oscillatory drainage forces between a sphere and a plane, suitable for dynamic SFA or Atomic Force Microscopy non-contact measurements. We use this model to investigate the boundary friction of viscoelastic polymer solutions over 5 decades of film thicknesses and one decade in frequency. The proper use of the original Navier's condition describes accurately the complex hydrodynamic force up to scales of tens of micrometers, with a simple "Newtonian-like" friction coefficient, not frequency dependent, and reflecting closely the dynamics of an interfacial depletion layer at the solution/solid interface.
\end{abstract}

ก 
Flow of complex liquids are familiar and useful. Unlike Newtonian fluids, they display complex bulk rheological behavior, non-linear and frequency-dependent. But the way they flow also involves their boundary conditions on solid surfaces. The boundary condition (b.c.) is relevant for small scale flows, occurring for instance in bio-medical applications, microfluidic devices, food or oil engineering, but also for the faithful characterization of the bulk rheology [1] [5].

As in the case of simple liquids, the slippage of complex fluids at walls is commonly characterized by a slip length $b$, defined by the ratio of the fluid velocity at the solid surface to the shear rate at the wall: $v_{\text {slip }}=b \partial v(z) / \partial z$, with $z$ the direction normal to the boundary. But the notion of slip length, now well established and understood in the case of simple fluid flowing on various types of solid surfaces [6] 12] or Newtonian polymer melts [13, 14, is far from being obvious in the case of more complex fluids. We show here experimentally that the appropriate quantity to describe the boundary slippage of complex fluid without ambiguity, is indeed not a slip length, but rather a liquid/wall friction coefficient, as originally stated by Navier [15].

We demonstrate this on the particular example of semi-dilute, viscoelastic poly-electrolyte solutions. Water-soluble polyelectrolytes of high molecular weight are commonly use to thicken water solutions at an affordable price, as small concentrations are sufficient to increase significantly the viscosity of the solution [16. Water-soluble polyelectrolytes of high molecular weight have been reported to display large slip on various types of solid surfaces [17-20. This large slip has been attributed to the presence of a depletion layer at the solution/solid interface, i.e. a layer with a lower concentration of polymer or even with pure solvant, whose viscosity significantly lower than that of the solution induces an apparent slip boundary condition [21 25. Here we use Partially Hydrolyzed PolyAcrylamide (HPAM) semi-dilute solutions, in conditions which were otherwise well characterized by other groups and are industrially used for Enhanced Oil Recovery or water purification 20] (SNF Flopaam 3630S, molecular weight 20.10 6 g/mole solved in deionized water at concentration from 0.8 to $1.6 \mathrm{~g} / \mathrm{l}$ ). The boundary flow of these solutions is studied with a dynamic Surface Force Apparatus (dSFA, 26, 27]) by confining them between a pyrex sphere (radius $R=3.3 \mathrm{~mm}$ ) and a pyrex plane of very low roughness $(2 \AA \mathrm{rms}$ as measured by AFM). The set-up covers sphere-plane distances $D$ ranging over 5 orders of magnitudes from $0.1 \mathrm{~nm}$ to $15 \mu \mathrm{m}$, allowing one to bridge the macroscopic flow behavior of the liquid to its interfacial hydrodynamics.
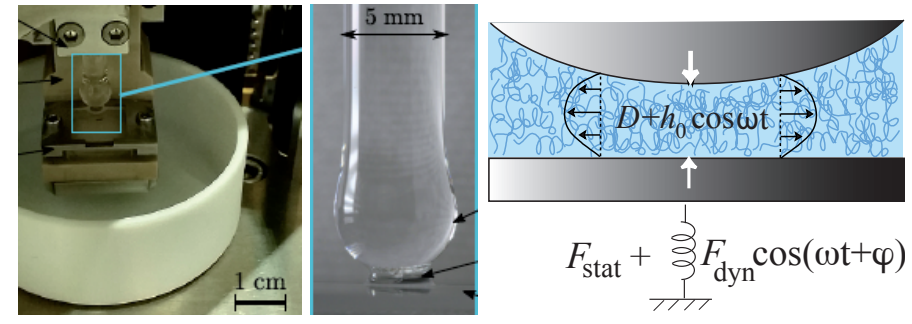

FIG. 1. The dynamic Surface Force Apparatus. Left: large view of the sphere-plane contact. Center: detailed view of the contact. Right: Schematic of the flow.

The sphere is driven normally to the plane at a low drift velocity $\left(\dot{D} / D<10^{-2} \mathrm{~s}^{-1}\right)$. An oscillatory motion of small amplitude $\left(h_{0} / D<10^{-2}\right)$ at angular frequency $\omega$ is added to the slow drift motion. The relative sphere-plane displacement as well as the force acting on the plane, are measured by two independent external interferometric sensors. From these measurements we get the steady-state sphere-plane distance $D$ and interaction force $F_{\text {stat }}$, the dynamic amplitude $h_{0}$ of the sphere-plane oscillatory displacement which is chosen as the phase origin, the dynamic amplitude $F_{\mathrm{dyn}}$ and phase-shift $\varphi$ of the oscillatory interaction force at the frequency $\omega$, and finally, the linear force response or mechanical impedance, defined as:

$$
\tilde{Z}(D, \omega)=\frac{F_{\mathrm{dyn}} e^{i \varphi}}{h_{0}}=Z_{R}+i Z_{I}
$$

For a viscoelastic liquid of complex shear modulus $\tilde{G}=G_{R}+\mathrm{i} G_{I}=\mathrm{i} \omega\left(\eta_{R}-\mathrm{i} \eta_{I}\right)$, the hydrodynamic force response in this oscillatory drainage flow, in the case of a no-slip boundary conditions at walls, is [28]:

$$
\tilde{Z}(D, \omega)=\frac{6 \pi R^{2} \tilde{G}}{D}=\frac{6 \pi R^{2} \mathrm{i} \omega \tilde{\eta}}{D}
$$

Therefore it is convenient to characterize the bulk viscoelasticity of the solutions by plotting $1 / Z_{\mathrm{R}}$ and $1 / Z_{\mathrm{I}}$ as a function of the sphere-plane distance, looking at large values of $D$ (fig 2). At large distance a well-defined linear behavior is observed, demonstrating the bulk viscoelastic character of the solutions. From the slope of $1 / Z_{R}$ and $1 / Z_{I}$ we extract the complex shear modulus components $G_{R}$ and $G_{I}$. 

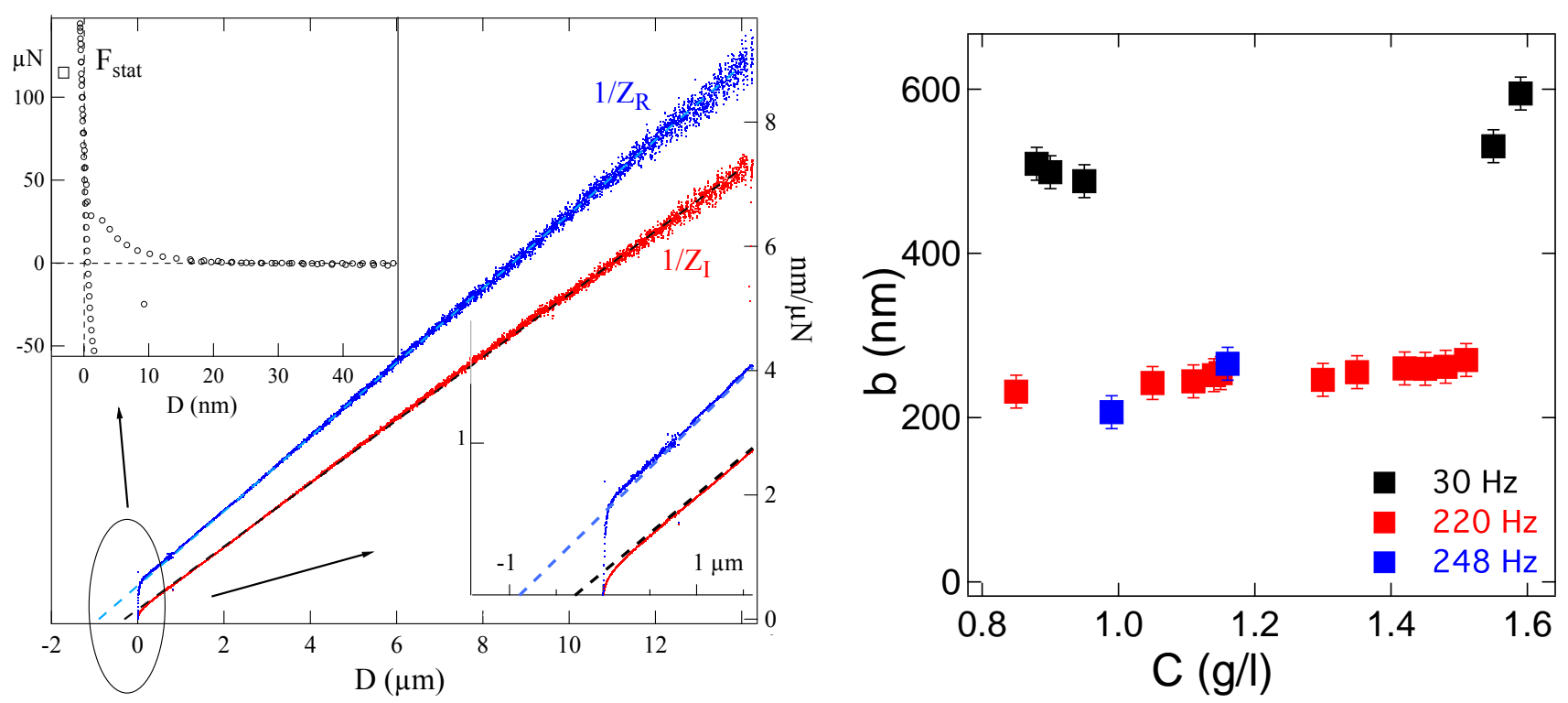

FIG. 2. Left: inverse of the components of the dynamic force response $\tilde{Z}$ measured in an HPAM solution at a frequency of 220 $\mathrm{Hz}$, as a function of the sphere-plane nominal distance $D$. Blue: $1 / Z_{R}$, red: $1 / Z_{I}$. The top-left inset shows the quasi-static interaction force $F_{\text {stat }}$, whose jump-to-contact defines the origin of distances. The down-right inset is an enlargement at the submicrometric scale. Right: slip length defined from the extrapolation length of $1 / Z_{I}$ (intercept with the $\mathrm{x}$-axis of the black dashed line of the left plot), as a function of the concentration of the solution, for various frequencies.

However, contrary to the prediction of eq. 2, the extrapolated far-field linear dependency of $Z_{R}^{-1}$ and $Z_{I}^{-1}$ does not point toward the distance origin, but towards some negative values of $D$. This is usually the signature of a slippage effect at the solid-liquid interface. More specifically, it is known in simple fluids that if a slip length $b$ defines the slip boundary condition on each surface then the hydrodynamic force at large distance $D \gg b$ involves the "hydrodynamic thickness" $D+2 b$ instead of the actual distance $D$. It is thus tempting to describe the measurements by deriving a slip length from the extrapolation lengths of $1 / Z_{R}$ and $1 / Z_{I}$. However when doing so, two difficulties appear. First, the two linear extrapolations actually point towards two different origins, which is not consistent with a single, well-defined slip length. Instead, the extrapolations tend to show that the slip length is complex, possessing a real and an imaginary components. Second, if one determines the slip length as in simple liquids from the extrapolation length of the damping $1 / Z_{I}$, one finds that it depends significantly on the frequency of the oscillatory flow (see fig. 2). At a concentration $\sim 1 \mathrm{~g} / \mathrm{l}$ for instance, the slip length decreases by a factor larger than 3 when the frequency increases from $30 \mathrm{~Hz}$ to $220 \mathrm{~Hz}$. This complex and frequency-dependent behavior of the slip length does not reflect the mechanism usually producing large slip of polymer solutions on solid surfaces, which involves the presence of an interfacial depletion layer made of pure solvant [23, 24]: in the presence of a purely Newtonian lubricating layer, one should expect a purely dissipative and fully Newtonian friction mechanism of the polymer solution onto the solid surface.

These two difficulties arise because the slip length $b$ is actually defined as the ratio $\eta / \lambda$ of the liquid viscosity to the interfacial friction coefficient first introduced by Navier's in its original statement of the boundary condition [15]:

$$
\lambda v_{\text {slip }}=\eta \frac{\partial v(z)}{\partial z}
$$

For Newtonian fluids the viscosity is a constant quantity, not dependant on the frequency or shear rate, and the slip length thus provides a convenient image of the interfacial friction. However the mixture of bulk and interfacial properties entering in the slip length definition raises ambiguities when the fluid is non-Newtonian.

Keeping this in mind, we proceed to extract directly the interfacial friction coefficient from the force measurements in oscillatory drainage flow experiments. For this purpose, we calculate the hydrodynamic force exerted by the fluid drainage between the sphere and the plane, when the viscoelastic fluid undergoes the Navier's partial slip boundary condition eq. (3) on both surfaces. We restrict to the linear response limit $h_{0} \ll D$, in which all time variations are harmonic at the forcing frequency $\omega$, and all time-varying quantities are characterized by their complex amplitude. In these conditions the (amplitude of the) stress tensor in the liquid is $\overline{\bar{\sigma}}=\tilde{\eta}\left(\nabla \overrightarrow{\tilde{v}}+{ }^{T} \nabla \overrightarrow{\tilde{v}}\right)-\delta \tilde{P} \overline{\bar{I}}$, where $\delta \tilde{P}$ is the 

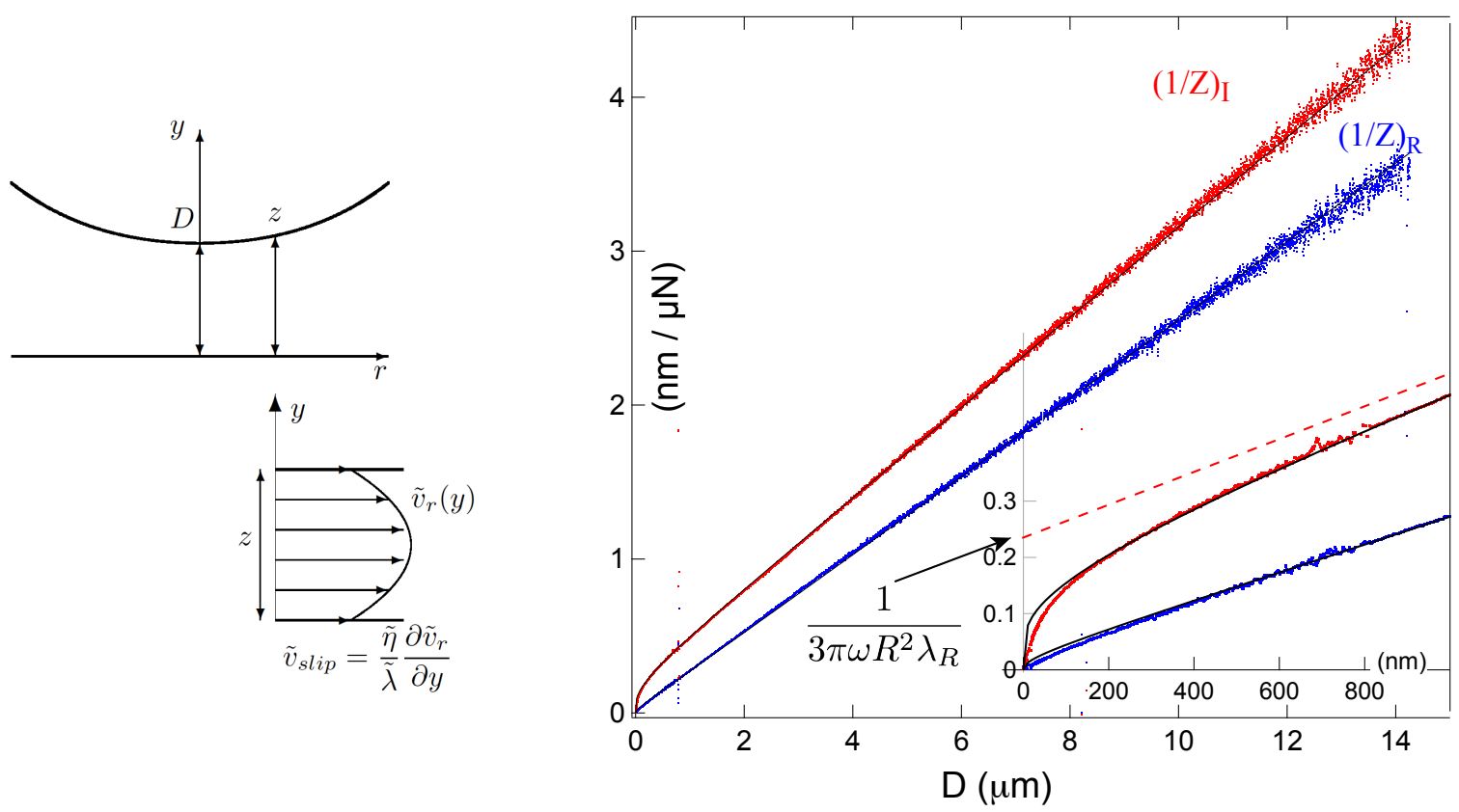

FIG. 3. Left: schematic of the sphere-plane geometry and of the flow profile. Right: elastic component $(1 / \tilde{Z})_{R}($ blue dots) and dissipative component $(1 / \tilde{Z})_{I}$ (red dots) of the inverse of the force response measured in an HPAM solution at a frequency of $220 \mathrm{~Hz}$. The black continuous lines are the components of the theoretical expression 8 fitted with a real-valued boundary friction coefficient $\tilde{\lambda}=\lambda_{R}$. Inset: enlargement below the micrometric scale. The red dashed line is the extrapolation of the far-field behavior of $(1 / \tilde{Z})_{I}$.

amplitude of the dynamic pressure inducing the hydrodynamic force

$$
\tilde{F}_{\mathrm{dyn}}=\int_{0}^{\infty} 2 \pi r \delta \tilde{P}(r) d r
$$

The Navier's boundary condition 3 is used with a complex friction coefficient $\tilde{\lambda}$. In a Maxwell model of the interface, $1 / \tilde{\lambda}=1 / \lambda_{R}+i / \omega k$, where $k$ is the interface stiffness and $\lambda_{R}$ the dissipative friction coefficient.

At small distance $D \ll R$, most of the hydrodynamic force originates from regions where the two solid surfaces are almost parallel. In these conditions, for angular frequencies such that $T=2 \pi / \omega \gg|\tilde{\eta}| / \rho R D$ inertia is negligible, and the average flow velocity is given by the lubrication approximation [29]:

$$
\tilde{u}(r)=\frac{1}{z} \int_{0}^{z} \tilde{v}_{r}(r, y) d y=-\frac{1}{12 \tilde{\eta}} \frac{d \delta \tilde{P}}{d r}\left(z^{2}+6 z \frac{\tilde{\eta}}{\tilde{\lambda}}\right)
$$

where $z(r)$ is the nominal gap between the surfaces at distance $r$ from the axis. Note that the fluid velocity and the dynamic pressure $\delta \tilde{P}$ are of first order in $h_{0}$, so that only the nominal gap $z(r)$, equal to $z=D+r^{2} / 2 R$ in the parabolic approximation, enters in (5). For rigid solid surfaces, the average velocity $\tilde{u}(r)$ obeys the conservation relation:

$$
\frac{d(2 \pi r z(r) \tilde{u}(r))}{d r}=-2 \pi r \frac{\partial z(r)}{\partial t}=-2 \pi r i \omega h_{0}
$$

Eq. (5) and (6) give the following equation for the pressure:

$$
\frac{d}{d r}\left[r z^{2}\left(z+\frac{6 \tilde{\eta}}{\tilde{\lambda}}\right) \frac{d \delta \tilde{P}}{d r}\right]=12 \mathrm{i} \omega r \tilde{\eta} h_{0}
$$

which is integrated twice with $r d r=R d z$ to obtain the hydrodynamic force:

$$
\begin{array}{r}
\tilde{Z}=\frac{\tilde{F}_{\mathrm{dyn}}}{h_{0}}=\frac{6 \pi R^{2} \mathrm{i} \omega \tilde{\eta}}{D} f^{*}\left(\frac{\tilde{\eta}}{\tilde{\lambda} D}\right) \\
f^{*}(\tilde{y})=\frac{1}{3 \tilde{y}}\left[\left(1+\frac{1}{6 \tilde{y}}\right) \ln (1+6 \tilde{y})-1\right]
\end{array}
$$



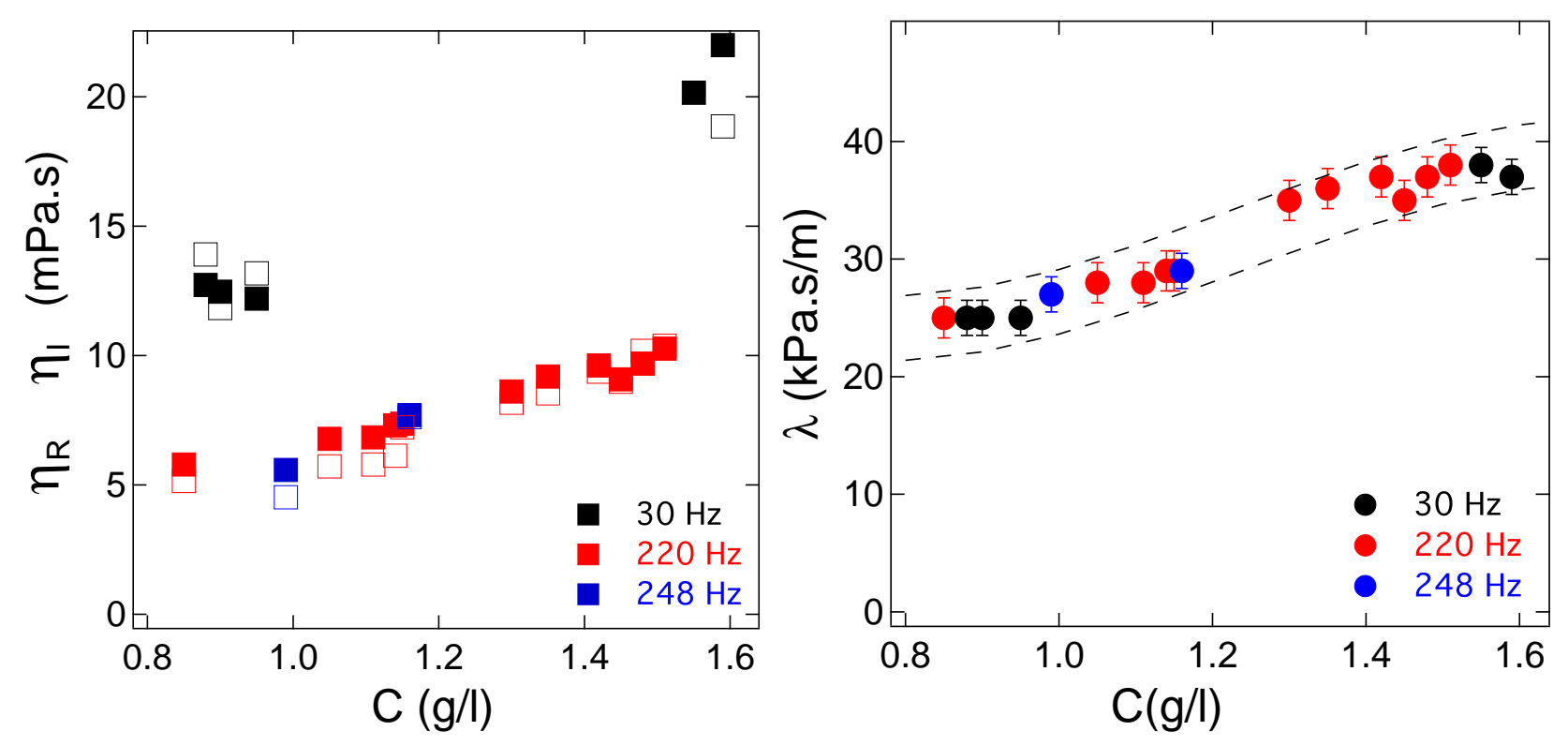

FIG. 4. Left: components $\eta_{R}(\mathbf{\square})$ and $\eta_{I}(\square)$ of the visco-elastic modulus $\tilde{\eta}$ of the solutions as a function of polymer concentration, at $30 \mathrm{~Hz}$ (black), $220 \mathrm{~Hz}$ (red), and $248 \mathrm{~Hz}$ (blue). Right: interfacial friction coefficient $\tilde{\lambda}=\lambda_{R}$ at the solution/solid boundary as a function of the HPAM concentration, at $30 \mathrm{~Hz}(\bullet), 220 \mathrm{~Hz}(\bullet)$, and $248 \mathrm{~Hz}(\bullet)$. The dashed lines are a guide for the eye.

Note that the logarithm entering eq. (9) should be calculated taking into account the complex character of its argument, by $\ln \left(r e^{i \theta}\right)=\ln r+\mathrm{i} \theta$.

Equations (8) and (9) generalize the Hocking expression [29] derived for a Newtonian liquid slipping on the solid surfaces with a slip length $b$. In the Hocking expression, the factor $f^{*}$ has the same mathematical expression as in eq. $(9)$, but it depends only on the simple ratio $D / b$. In the present non-Newtonian case, we see that a "complex slip length" $\tilde{b}=\tilde{\eta} / \tilde{\lambda}$ governs the hydrodynamic force, which explains the two extrapolation lengths observed in figure (2). The complex character of the slip length reflects the phase difference between the boundary slip velocity and the bulk velocity, and the ratio $\tilde{b} / D$ reflects the impact in amplitude and phase of the wall slippage in the bulk flow. We can see that for a viscoelastic liquid the slip length $\tilde{b}$ is complex even if the interfacial friction is purely dissipative, which suggests that the complex frequency-dependent slip length observed above might be only an artifact due to the bulk behavior of the solutions.

In order to disentangle experimentally the interfacial boundary condition from the bulk properties of the solutions we notice that at large distance $D \gg|\tilde{\eta} / \tilde{\lambda}|$, the above expression $(8)$ expands as:

$$
\frac{1}{\tilde{Z}}=\frac{D}{6 \pi R^{2} \mathrm{i} \omega \tilde{\eta}}+\frac{1}{3 \pi \mathrm{i} \omega R^{2} \tilde{\lambda}}
$$

Thus, rather than $Z_{R}^{-1}$ and $Z_{I}^{-1}$, the dynamic quantity providing an independent access to the interfacial rheology is $1 / \tilde{Z}$. More specifically, the boundary friction and stiffness are obtained from the value at origin (intercept on the $\mathrm{y}$-axis) of the linear extrapolation of the far-field components $(1 / \tilde{Z})_{R}$ and $(1 / \tilde{Z})_{I}$. Accordingly, the components of $1 / \tilde{Z}$ in our HPAM solutions are plotted in fig 3 . The linear extrapolation of $(1 / \tilde{Z})_{R}$ points towards the origin within the experimental resolution. This shows that elastic effects in the interface response are negligible: $k \simeq 0$ and $\tilde{\lambda}$ reduces to $\lambda_{R}$. The purely dissipative nature of the interfacial friction coefficient reflects the physical mechanism inducing the apparent slip, i.e. the lubrication effect of a Newtonian liquid layer at the boundary.

The linear extrapolation of $(1 / \tilde{Z})_{I}$ (dashed red line fig 3 does not extrapolate to zero and gives a first estimation of the liquid/solid friction coefficient $\lambda_{R}$, in the range of $30 \mu \mathrm{Pa} . \mathrm{s} / \mathrm{nm}$. For a precise determination of $\lambda_{R}$ it is however important to compare the whole data to the theoretical expression (8, 9), because the asymptotic linear dependency of $1 / \tilde{Z}$ with $D$ is reached only at very large distances $D \gg|\tilde{\eta}| /|\lambda|$. At smaller distances, $(1 / \tilde{Z})_{I}$ curves down continuously, converging finally towards the physical origin. This tendency is in excellent agreement with our theory which provides a very accurate prediction of both components of the measured dynamic force (see figure 3 ).

Precise estimations of the boundary friction coefficient $\lambda_{R}$ are obtained for various concentrations and frequencies by fitting the data to eq. 8 , and are plotted in figure 4. Unlike the slip length (fig. 2 the friction coefficient is 


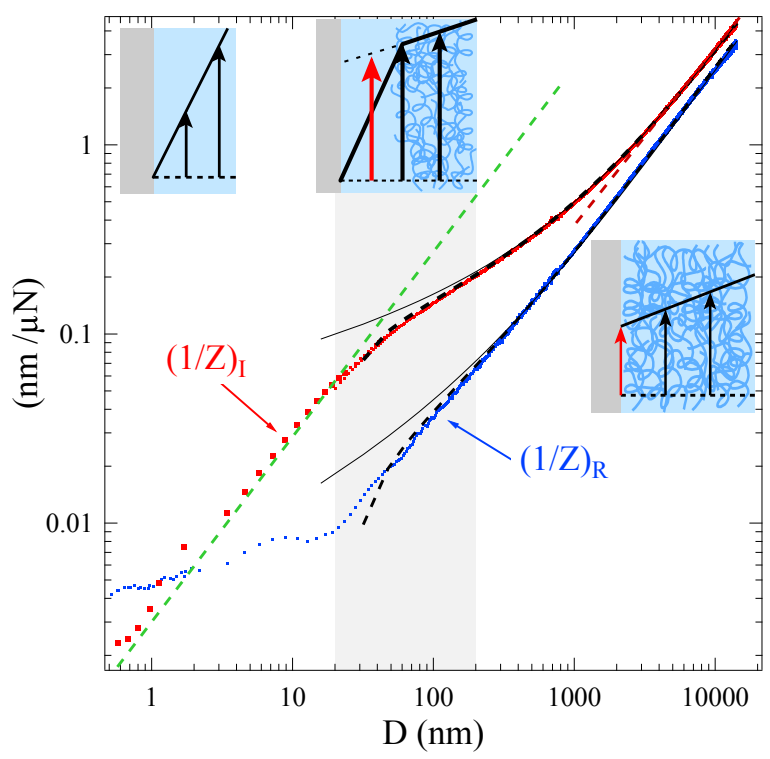

FIG. 5. Log-log plot of the components of $1 / \tilde{Z}$ (blue dots: real, red dots: imaginary) in a solution of $1.4 \mathrm{~g} / \mathrm{l}$ at $220 \mathrm{~Hz}$, showing the macro-micro transition. The dashed red (resp. green) line corresponds to a Newtonian fluid (resp. water) with a no-slip b.c. at the solid wall. The black continuous lines are the components of the theoretical expression (8) taking into account the bulk viscoelasticity and a Navier's b.c. with a real-valued friction coefficient $\lambda_{R}$. The dashed continuous lines are eq. 8) with the boundary condition applied inside the liquid, at a distance $e_{s} / 2=\eta_{\text {water }} / 2 \lambda_{R}=14 \mathrm{~nm}$ of each solid surface.

found essentially insensitive to the frequency over the range studied, which extends on one decade. The absence of frequency evolution is the signature of a fully Newtonian interfacial friction, as expected for a slippage mechanism involving a fully depleted, pure water layer at the solution/solid interface. In contrast, the viscoelastic modulii of the solutions vary significantly in the range of frequencies studied (see figure 4 left). This variation accounts fully for the frequency-dependance of the slip length measured in figure 2, which is thus due to the bulk rheology of the solutions, and not to the boundary hydrodynamics. Assuming that the viscosity of the depletion layer is that of pure water, its estimated thickness $e_{s} \simeq \eta_{\text {water }} / \lambda$ [30, 31 varies between $40 \mathrm{~nm}$ to $26 \mathrm{~nm}$ for a bulk concentration of polymer between 0.8 to $1.6 \mathrm{~g} / \mathrm{l}$. Furthermore, we should emphasize that in dynamic SFA experiments, the wall shear rate is not spatially uniform, and its range of values changes when the nominal distance $D$ is varied. Considering the excellent agreement of our analytical expression with the data, we can state that the boundary friction coefficient is insensitive to the flow geometry, frequency, and shear rate at wall in the range of $10^{-5}$ to $10^{-2} \mathrm{~s}^{-1}$ probed in the experiment. This shows that the boundary layer inducing the apparent slip is a purely equilibrium layer, whose properties are independent on the applied flow in the range of the above frequencies and shear rates. Such a conclusion cannot be obtained from the properties of the slip length alone, which provides a further proof that the liquid/solid friction coefficient is more appropriate than the slip length to characterize the boundary slip of a complex fluid.

Finally, we discuss the limitations at the microscopic scale of the model of apparent slip boundary condition. The connection of the macroscopic scale to the microscopic scale is shown in figure 5 . Three areas appear on this figure. Below 20nm, the depletion layer at the solution/solid interfaces is evidenced by the behavior of the damping $(1 / \tilde{Z})_{I}$ : the latter cannot be distinguished from a Newtonian liquid of viscosity $0.85 \mathrm{mPa} . \mathrm{s}$, essentially equal to that of water at the experiment temperature $\left(28^{\circ} \mathrm{C}\right.$ ), flowing with a no-slip boundary condition on the solid surfaces (green dashed line in figure 57. The microscopic scale of $20 \mathrm{~nm}$ is indeed in good agreement with the estimated thickness $e_{s} \simeq$ $28 \mathrm{~nm}$ of the depletion layer at this polymer concentration of $1.4 \mathrm{~g} / \mathrm{l}$. Thus at distances $D$ smaller than $e_{s}$, the sphere-plane gap is largely filled with pure water and the hydrodynamic force resumes to the Reynolds force with a no-slip boundary condition located onto the solid surfaces. Second, at intermediate distances $e_{s}<D<10 e_{s}$, the polymer solution flows on a lubricating layer whose thickness cannot be neglected. Accordingly, as shown theoretically [7, the apparent b.c. has to be properly described by 2 independent parameters: the location of the b.c. and the interfacial friction coefficient. For a sharp and large viscosity gradient the appropriate apparent b.c. location lies close to the middle of the depletion layer $e_{s}$ as sketched in fig. (5). Taking this location for the b.c. instead of the actual position of the solid surfaces, amounts to replace $D$ by $D+e_{s}$ in equations (8, 9). We find that it provides a significantly better agreement with the data (dashed black lines in figure 5). Third, at large distances $D>10 e_{s}$, the finite thickness of the depletion layer can be neglected and one retrieves the apparent slip boundary condition 
characterized by a friction coefficient $\tilde{\lambda} \approx \lambda_{R}=\eta_{\text {water }} / e_{s}$.

In conclusion, we have shown that the slip behavior of a complex fluid at a solid boundary should be consistently analyzed using a boundary friction coefficient rather than a slip length, in order to account faithfully for interfacial rheology effects. In the studied case, the friction coefficient is real-valued and fully Newtonian while the slip length $\tilde{\eta} / \lambda$ is complex and frequency dependent, due to the complex bulk rheology of the solutions. This fully Newtonian friction reflects a slip mechanism due to a depletion layer made of pure solvent at the solid-polymer solution interface. Our experiments and analysis show that, beyond a proper description of the boundary condition, the friction coefficient is the relevant quantity for understanding the physical mechanisms governing the interfacial dynamics. From a fundamental point of view, the coupling between the generalized lubrication theory developed here and the unique capabilities of the SFA, opens the route to develop a complete surface rheology for the solid-liquid interface that could be equivalent to what has been recently developed for the fluid-fluid interface [32. In particular it would be interesting to perform experiments on interfaces specifically tailored at the molecular level to provide a non-Newtonian surface friction. Polymer systems are choice candidates to do so.

This research was supported by the ANR program ANR-15-CE06-0005-02.

[1] Howard A. Barnes, "A review of the slip (wall depletion) of polymer solutions, emulsions and particle suspensions in viscositmer: its cause, character, and curve," J. Non-Newtonian Fluid Mech. 56, 221-251 (1995).

[2] M.M. Denn, "Extrusion instability and wall slip," Annu. Rev. Fluid Mech. 33, 265-287 (2001).

[3] G. Reiter and R. Khanna, "Real-time determination of the slippage length in autophobic polymer dewetting," Phys. Rev. Letter 85, 2753 (2000).

[4] O. Baumchen and K. Jacobs, "Slip effects in polymer thin films," J. Phys. Cond. Mat. 22, 033102 (2010).

[5] Savvas G. Hatzikiriakos, "Wall slip in molten polymers," Progress in Polymer Science 37, 624-643 (2012)

[6] P.A. Thompson and M.O. Robbins, "Shear flow near solids: Epitaxial order and flow boundary conditions," Phys. Rev. A 41, 6830 (1990).

[7] L. Bocquet and J.-L. Barrat, "Hydrodynamic boundary conditions, correlation functions, and kubo relations for confined fluids," Phys. Rev. E 49, 3079-3092 (1994).

[8] P.A. Thompson and S.M. Troian, "A general boundary condition for liquid flows at solid surfaces," Nature 389, 360-362 (1997).

[9] R. Pit, H. Hervet, and L. Léger, "Direct experimental evidence of slip in hexadecane : solid interfaces," Phys. Rev. Lett. 85, 980-983 (2000).

[10] L. Thomas, E. Charrault, and C. Neto, "Interfacial slip on rough, patterned and soft surfaces: A review of experiments and simulations," Adv. in Coll. and Interf. Sci. 210, 21-38 (2014).

[11] E. Secchi, S. Marbach, A. Niguès, D. Stein, A. Siria, and L. Bocquet, "Massive radius-dependent flow slippage in carbon nanotubes," Nature $\mathbf{5 3 7}$ (2016).

[12] S. Leroy, A. Steinberger, C. Cottin-Bizonne, F. Restagno, L. Léger, and E. Charlaix, "Hydrodynamic interaction between a spherical particle and an elastic surface: A gentle probe for soft thin films," Phys. Rev. Lett. 108, 264501 (2012).

[13] O. Baumchen and K. Jacobs, "Slip effects in polymer thin films," Journal Of Physics-Condensed Matter 22,-(2010).

[14] Marceau Hénot, Alexis Chennevière, Eric Drockenmuller, Liliane Léger, and Frédéric Restagno, "Comparison of the slip of a PDMS melt on weakly adsorbing surfaces measured by a new photobleaching-based technique," Macromolecules , $5592-5598(2017)$

[15] C.L. Navier, "Mémoires sur les lois du mouvement des fluides," Memoires de l'Académie des Sciences 6, 389-416 (1823).

[16] A.V. Dobrynin and M. Rubinstein, "Theory of polyelectrolytes in solutions and at surfaces," Prog. in Polymer Science 30, 1049-1118 (2005).

[17] Guy Chauveteau, M Tirrell, and A Omari, "Concentration dependence of the effective viscosity of polymer solutions in small pores with repulsive or attractive walls," Journal of colloid and interface science 100, 41-54 (1984).

[18] A Omari, M. Moan, and G. Chauveteau, "Wall effect in the flow of flexible polymer solution through small pores," Rheolocica Acta 28, 520-526 (1989).

[19] J. Cayer-Barrioz, D. Mazuyer, A. Tonck, and E. Yamaguchi, "Drainage of a wetting liquid: Effective slippage or polymer depletion?" Tribo. Lett. 32, 81-90 (2008).

[20] A. Cuenca and H. Bodiguel, "Submicron flow of polymer solutions: Slippage reduction due to confinement," Phys. Rev. Lett. 110, 108304 (2013).

[21] P. G. De Gennes, "Polymer solutions near an interface. adsorption and depletion layers," Macromolecules 14, 1637-1644 $(1981)$

[22] Howard A Barnes, "A review of the slip (wall depletion) of polymer solutions, emulsions and particle suspensions in viscometers: its cause, character, and cure," Journal of Non-Newtonian Fluid Mechanics 56, 221-251 (1995).

[23] T.L. Kuhl, A.D. Berman, S.W. Hui, and J.N. Israelachvili, "Part 1. direct measurement of depletion attraction and thin film viscosity between lipid bilayers in aqueous polyethylene glycol solutions," Macromolecules 31, 8250-8257 (1998). 
[24] R.G. Horn, O.I. Vinogradova, M.E. Mackay, and N. Phan-Thien, "Hydrodynamic slippage inferred from thin film drainage measurements in a solution of nonadsorbing polymer," J. Chem. Phys. 112 (2000).

[25] W. Knoben, N. A. M. Besseling, and M. A. Cohen Stuart, "Direct measurement of depletion and hydrodynamic forces in solutions of a reversible supramolecular polymer," Langmuir 23, 6095-6105 (2007)

[26] F. Restagno, J. Crassous, E. Charlaix, C. Cottin-Bizonne, and M. Monchanin, "A highly sensitive dynamic surface force apparatus for nanorheology," Rev. of Sci. Instr. 73, 2292-2297 (2002).

[27] L. Garcia, C. Barraud, C. Picard, J. Giraud, E. Charlaix, and B. Cross, "A micro-nano-rheometer for the mechanics of soft matter at interfaces," Rev. of Sci. Instr. 87, 113906 (2016).

[28] E. Pelletier, J.-P. Montfort, and F. Lapique, "Surface force apparatus and its application to nanorheological studies," Journal of Rheology 38, 1151-68 (1994).

[29] L.M. Hocking, "The effect of slip on the motion of a sphere close to a wall and of two adjacent spheres," J. of Engineering Mathematics 7, 207-221 (1973).

[30] Olga I. Vinogradova, "Drainage of a thin liquid film confined between hydrophobic surfaces," Langmuir 11, 2213-2220 (1995).

[31] Remco Tuinier and Takashi Taniguchi, "Polymer depletion-induced slip near an interface," Journal of Physics: Condensed Matter 17, L9-L14 (2005)

[32] M. Brenner and S. Howard, Interfacial transport processes and rheology (Elsevier, 2013).

Author Information Correspondence and requests for materials should be addressed to Elisabeth.Charlaix@univgrenoble-alpes.fr. 